tions can be explained easily by the hypothesis of Ramsey, explaining the mantle-core boundary by a phase-transition of material.

A curious correlation was shown also between geoid undulations and heat-flow distribution ${ }^{4}$, though this correlation is less tight. While the former correlations refer to deep connexions, the latter may be based only on shallow effects.

The authors ${ }^{4}$ suggest for this correlation an explanation by convection currents. Convection currents have, however, also tectonical effects. which must be reflected in the major tectonic features of the Earth. But it is hard to discover any relation between heat-flow 'undulations' and the tectonic zones of the Earth.

I wish first of all to direct attention to the connexions between core and geoid undulations probably being the keys of problems far from being solved.

Geophysical Institute,

L. EGYED

Eötvös University,

Budapest, Hungary.

${ }^{1}$ Cook, A. H., Nature, 198, 1186 (1963).

' Izsák, J. G., Nature, 198, 137 (1963).

${ }^{3}$ Vogel, A., Gerl. Beitr. zur Geophys., 69, 150 (1960).

'Lee, W. H. K., and MacDonald, G. J. F., J. Geophys. Res., 68, 6481 (1963).

\section{Periodic Precipitation of the Particle Flux in the Auroral Zone}

EVIDENCE of periodic and quasi-periodic precipitation of the particle flux in the auroral zone is often observed in the riometer absorption ${ }^{1}, 5577 \AA$ photometer records, visual observation of pulsating aurora (also seen in the all-sky camera records) and other related geophysical phenomena. Further, the recent satellite observations have also shown periodic variation in the measured flux ${ }^{2}$. In this communication a possible mechanism for the periodic precipitation of the particle flux is proposed and its validity and limitations are discussed.

It is well known from the work of Dessler ${ }^{3}$, Piddington ${ }^{4}$ and of Dessler and Parker ${ }^{5}$ that the compressional mag. netic disturbance travels along the magnetic field as a transverse hydromagnetic wave. During this period longitudinal acoustic wave propagates perpendicular to the magnetic field ${ }^{6}$. Herlofson ${ }^{7}$ pointed out that in the presence of a magnetic field the longitudinal acoustic wave propagates with hybrid velocity and is known as magnetic-acoustic wave ${ }^{8}$. Its velocity is :

$$
V_{\mathrm{ma}}=\sqrt{V_{\mathrm{a}}^{2}+V_{\mathrm{hm}}^{2}}
$$

where $V_{\mathrm{ma}}, V_{\mathrm{a}}$ and $V_{\mathrm{hm}}$ are magneto-acoustic, acoustic and hydromagnetic velocities.

The incident solar plasma excites a longitudinal magneto-acoustic wave in the finite equatorial crosssection of the Van Allen belt, causing a compression and rarefaction in the intervening plasma and in the magnetic field. The magneto-acoustic waves propagating along the oquatorial belt are heavily damped at high frequencies and are refracted away from the equatorial region. However, under idealized conditions such propagation at low frequencies (smaller than the proton gyro-frequency) is a possibility, and was pointed out by MacDonald ${ }^{\circ}$. The compression and the rarefaction in the magnetic field result in a one-dimensional compression and rarefaction of the intervening plasma. During compression, diffusion of the plasma along the magnetic field is comparatively more than the diffusion perpendicular to the magnetic field. Consequently, a larger number of particles will have required pitch angle $\left(\leqslant 2^{\circ}\right)$ to be precipitated into the auroral zone. On the other hand during the rarefaction of the magnetic field, lesser numbers of particles will have required pitch angle to be precipitated into the auroral zone. The periodicity thus produced will have the period of the magneto-acoustic wave propagating along the equatorial Van Allen belt.

The phenomena may further be complicated if there exists a coupling of the transverse hydromagnetic waves along the field lines and the longitudinal magnetoacoustic wave along the equatorial belt. The resonance between the two modes propagating perpendicular to each other will occur only when the fundamental period of one is equal to, a multiple of, or sub-multiple of the other. In this situation, particle flux heading to the lower ionosphere (having required pitch angle) is greatly affected. The period of the incident flux on the ionosphere in this case is governed by the frequency of resonance between the transverse hydromagnetic wave and longitudinal magneto-acoustic wave propagating approximately perpendicular to each other.

An approximate estimate of the fundamental period of the longitudinal magneto-acoustic wave along the equatorial belt in the presence of the magnetic field is given by:

$$
T=\frac{2 \pi R_{E}}{\sqrt{V_{\mathrm{a}}^{2}+V_{\mathrm{hm}}^{2}}}
$$

Taking average value of the proton mass density obtained from nose whistler data ${ }^{10}$ :

$$
\rho=1.2 \times 10^{-2} \frac{B_{0} e m_{i}}{2 \pi e m_{e}}
$$

the period

$$
T=0 \cdot 28 \pi R_{E}^{5 / 2}\left(\frac{e m_{i}}{a m_{e}}\right)^{1 / 2}
$$

where $m_{i}, m_{e}$ are the proton and electron mass respectively, $e$ is the electron charge, $R_{E}=5.4$ equatorial radial distance and $a=8.1 \times 10^{25}$ gauss $\mathrm{cm}^{3}$ is the dipole moment of the Ear $\mathrm{h}$. The fundamental period from equation (2) becomes approximately 300 sec, which is equal to the most probable period reported by Parthasarathy and Hessler ${ }^{1}$. This period mainly depends on the exact knowledge of the proton mass density in the region of magneto-acoustic propagation. The periods of the transverse hydro-magnetic waves obtained from the micro-pulsations at mid-latitude ${ }^{11}$ and from the spectral analysis of telluric currents ${ }^{12}$ have shown the composite nature of the observed period. At higher latitude, periods anywhere between 100-500 sec having different power may exist. Under this situation there are better chances for the magneto-acoustic wave to become coupled to a suitable transverse hydromagnetic wave and give rise to a resonance effect.

The narrow latitude coverage of the observed periodicity and its simultaneous and similar appearance seen in Macquarie Island, the riometer records from which are approximately magnetically conjugate to College, is in accordance with the proposed mechanism.

I thank R. Parthasarathy and Keith B. Mather for their advice. This work was supported in part by the National Aeronautics and Space Administration Contract No. $N A S 5-3595$ and U.S. National Science Foundation grant GP-947.

Geophysical Instituto,

University of Alaska, College, Alaska.

1 Parthasarathy, R., and Hessler, V. P., J. Geophys. Res. (submitted). ${ }^{2}$ Judge, D. L., and Coleman, jun., B. J., J. Geophys. Res., 67, 5071 (1962). ${ }^{3}$ Dessler, A. J., J. Geophys. Res., B3, 405 (1958).

- Piddington, J., J. Geophys. Roy. Astro. Soc., 3, 173 (1959).

s Dessler, A. J., and Parker, E. N., J. Geophys. Res., 64, 2239 (1959).

- Dungey, T. W., Cosmic Electrodynamics, 75 (Cambridge Univ. Press, 1958).

"Herlofson, N., Nature, 165, 1020 (1950).

s De, J., Phys. of Fluids, 6, 1772 (1963).

MacDonald, G. J. F., J. Geophys. Res., 66, 3639 (1961).

${ }^{10}$ Smith, R. L., J. Geophys. Res., 66, 3709 (1961).

${ }^{11}$ Ellis, G. R. A., Austral. J. Phys., 13, 625 (1960).

${ }^{12}$ Mather, K. B., Gauss, E. J., and Cresswell, G. R., Austral. J. Phys. (submitted). 